\title{
The philosophy of Emergence and Complexity in the service of Osteopathy: in search of an epistemology of osteopathy in dialogue with contemporary philosophy.
}

\author{
Jean Fiore ${ }^{1, *}$ and Marjolaine Bouaissier ${ }^{2}$. \\ ${ }^{1}$ E.C.O., Centre Interdisciplinaire d'Ethique, 23 place Carnot - 69002 Lyon, France. \\ ${ }^{2}$ E.C.O., Centre Interdisciplinaire d'Ethique, 23 place Carnot - 69002 Lyon, France.
}

\begin{abstract}
Osteopathy is a discipline of healthcare that calls for systemic handling of patients. Osteopathy research has struggled to provide proof of the effectiveness of this practice. We think that osteopathy research needs to evolve in order to find a methodology that would allow it to account for its specificities and originality. This article notes the contribution of the philosophy of emergence in the elaboration of an epistemology suited to osteopathy as the precondition for a rationality adapted to the singular nature of this form of therapy.
\end{abstract}

\section{Introduction}

Osteopathy is a key player today with regard to healthcare offered in Europe and particularly in France. Millions of patients consult osteopaths every year, and this is due to the tangible results of this therapy. This success is the fruit of a rigorous learning process giving access to a wide variety of treatment tools and a capacity for listening that is consistent with the holistic claims of our profession. In reality osteopathy is necessarily empirical, since it consists of the application of general rules in response to an individual situation that simultaneously depends on a patient and a therapist. The capacity for adaptation to the singularity of living matter is a rich resource for osteopathy, but it contributes to making this discipline a complex one. The consequence of this complexity is that it is difficult to produce scientific evidence that would allow such a practice to be legitimized. In the following presentation, we will examine this complex nature and try to identify a model of thought that allows the particularities of this discipline to be taken into account.

Osteopathy was born in the United States under the leadership of A.T. Still (1828-1917). In his time Still founded osteopathy in order to propose an alternative form of healthcare that he considered more rational than the heroic medicine he opposed, which was the most widespread system of medical thought in the United States. Even if he probably had not read Claude Bernard, Still considered that medicine should be experimental, starting from clinical observation, and that it should constantly question its own practices in order to eliminate from them everything that is useless and dangerous.
The fundamental principles of the practice shaped by Still were systematized in the Kirksville consensus declaration of 1953. Today we can summarize them as follows: the interrelation between structure and function, homeostasis, the importance of the good circulation of fluids and the unity of the human being. From the outset Still was aware of the complexity of the medical approach he was creating, and even if training is based on theoretical knowledge such as that of anatomy and physiology, which fall within a reductionist approach, perception is immediately at the centre of osteopathic practice. This particular perception can be learnt, but ideally, the teaching of osteopathic perception is not so much about learning what is perceived as it is about how to perceive. The learning of osteopathy is therefore structured in terms of two registers: that of acquiring knowledge, and that of perceptual experience, which is individual and specific to each osteopath. It follows that the patient-therapist relationship in osteopathy is an experience that is more about intelligent co-creation than about reproducing of a protocol. The evaluation of osteopathy by the tools of pharmaceutical medicine alone is therefore impossible, and when it is tried, such an attempt necessarily results in mutilation.

After first presenting osteopathy in order properly to grasp its complexity, we will insist on the issues involved in its recognition. This necessity for legitimation requires the construction of an epistemology specific to osteopathy; seen from this angle we will examine the potential of the philosophy of emergence, which is a philosophy of complexity.

\footnotetext{
* Corresponding author : fiore.osteo@posteo.net
} 


\section{Presentation of the complex aspect of osteopathy}

Knowing the patient in osteopathy therefore requires several levels of understanding. There is the knowledge shared by patients regarding their own bodies, as they live them, and which will be compared with the theoretical knowledge and clinical experience of the osteopath. There is the anatomical-physiological reading that the osteopath makes when she interacts with the patient's body, and finally there is the knowledge that the patient's body has its of own functioning or dysfunction, and this knowledge is a corporeal intelligence which is not necessarily cognitive. Jean-Marie Gueullette is a medical doctor and historian; he has taken a particular interest in osteopathy in recent years, and he reminds us that "viewed from this standpoint, osteopathic perception does not consist of looking for signs interpreted by the intellect according to acquired patterns of understanding, but of a form of intelligent listening to knowledge possessed by the patient's body, even if the patient is no longer able to make use of it [1] (p. 70)".

Here we can already observe the construction of a discipline of healthcare that balances the principles (which, once assimilated, will be left in the background), and experience (which remains elusive because it occurs over time). A balance, therefore, between principles and experience, and which results in far greater consistency than principles on the one hand and experience on the other.

It seems to me that osteopathy succeeds in this tour de force of combining, within the same concept of healthcare, a theoretical basis which is based on reductionist physicalism, while accepting that a substantial part of the practice in which we are experienced cannot be described with the tools of reductionism alone.

But what is osteopathy? Here is the definition given by the WHO: "Osteopathy (also called osteopathic medicine) relies on manual contact for diagnosis and treatment. It respects the relationship of body, mind and spirit in health and disease; it lays emphasis on the structural and functional integrity of the body and the body's intrinsic tendency for self-healing.

Osteopathic practitioners use a wide variety of therapeutic manual techniques to improve physiological function and / or support homeostasis that has been altered by somatic (body framework) dysfunction, i.e. impaired or altered function of related components of the somatic system; skeletal, arthrodial and myofascial structures; and related vascular, lymphatic, and neural elements.

Osteopathic practitioners use their understanding of the relationship between structure and function to optimize the body's self-regulating, self-healing capabilities. This holistic approach to patient care and healing is based on the concept that a human being is a dynamic functional unit, in which all parts are interrelated and which possesses its own self-regulatory and self-healing mechanisms." [2]
The term manual therapy refers to the fact that it is mainly through her hands that the osteopath perceives and treats. And the osteopathic approach indeed accords great importance to palpation, allowing differences in temperature, consistency, density, and mobility to be recognized. The perceptual approach of the osteopath does not stop there, however, and it is with all their being that osteopaths enter into knowledge of the patient they are treating. Firstly because in the diagnostic phase, osteopaths do not only trust their hands, they use all of their perceptive abilities, they observe patients' behaviour, listen to their way of speaking; they can perceive smells, in short they are receptive to all sorts of information through their whole body and all their senses, not only via their hands. In addition, some osteopaths are led to perceive differently because they develop a form of receptivity, a receptivity requiring a free and stable interior state. In this type of osteopathy, the therapist will not be able to perceive anything if she does not perceive herself first; she will not be able to free herself from information coming from her own body if she is not present to herself. This particular interior disposition allows the osteopath to be prepared to receive what presents itself without having imagined it a priori a disposition conducive to the reception of novelty. [1] (pp. 59-68)

This having been said, the work of the osteopath can be seen as a way to refresh the patients' ability to be what they are becoming, and this by drawing their attention to what has become blocked in them. This attention is a form of setting in motion, tending towards a state of consciousness of being open to oneself.

The osteopath is often presented as a body mechanic who pulls, pushes, aligns the bones, the viscera, who relaxes muscles and ligaments. This is all correct, but what is played out in osteopathic sessions goes further. It can happen that, when working on a visceral tension, the osteopath perceives an emotion which does not seem linked to her own experience, and that at the same moment the patient decides to share their feelings which turn out to be in agreement with that of the osteopath, similar to an "impression". It can happen that in the middle of a session the patient will burst into tears or explode with laughter without understanding the origin of this manifestation, and it can be that this corresponds precisely to the moment when I as a therapist feel the relaxation of the tension on which I was working.

These astonishing perceptual experiences raise a question: in concrete terms, how can we think of what eludes explanation? The repetition of these perceptual events and their efficiency in treating patients has allowed me to accept that these are not the products of my imagination: no, we are really talking about effects produced by reality and which carry meaning. However, although perception is something obvious for me at the moment when I perceive it, it is not possible to justify scientific discourse solely on the basis of personal experience.

In reality, the osteopath's approach stems from a complex practice. First and foremost, it is a way of thinking in the area of health which is above all sensitive to mutual links, implications and interactions [1] (p.181). 
Osteopathy can be compared to an "art of complexity" which puts to work the capacity of a complex being, the patient, to adapt to the reality in which they are developing and with which they interact. In fact, more specifically, it is a question of the interaction of two complex systems - the therapist's sensing and perceiving body, and the sensed and perceived body of the patient, which themselves form a larger system.

\section{Issues of recognition and legitimacy}

As we have mentioned above, osteopathy is a complex practice. Today, when the practice of osteopathy is questioned, the issue of the legitimacy of its know-how is put in parallel with its capacity to provide proof of its effectiveness. J.M. Gueullette has done much work in this regard on the question of the relationship of osteopathy to medical science, and in his book L'ostéopathie, une autre medicine (Osteopathy, another form of medicine), he asks the following question: "What will the response of osteopaths be to these formal demands for the development of forms of research that are reliable? Will they have to modify their practices in order to allow them to fit within the methods of medical research, or will they be able to imagine suitable models?"[1] (p. 130).

Our E.C.O (Emergence, Complexity, Osteopathy) research group was born of the desire of several osteopaths to develop tools adapted to thinking about osteopathy. And we indeed think that it is urgent to equip osteopathy with its own epistemology. The urgency of this endeavour is underscored by what we are observing within our profession regarding a current of thought which considers that we must constrain the reality of our practice to reduce it to a set of techniques capable of justification by an experimental evaluative approach based on reductionism [3]. Some authors following this line of thought dismiss osteopaths by describing on the one hand a progressive osteopathy their own - that could be described as reductionist, while regarding the rest of the profession as traditionalist, backward-looking, practising a sort of vitalist osteopathy.

Rather than putting reductionism and vitalism in opposition, which are two different conceptions in the philosophy of science, and asking osteopaths to make a default choice, we propose adopting a mediating position between these two classic opposites. The philosophy of emergence belongs to this alternative path. Indeed, this philosophy offers a specific form of rationality, with an epistemic dimension, for thinking about complex phenomena. And the whole difficulty of emergence lies in its ability to encompass what at first sight appears to be a contradiction. It rejects both the body-mind dichotomy of vitalism and strict identity as in ontological materialism.

We will now go a little deeper into this singular position which seems to us to make emergence a particularly interesting concept for studying osteopathy.

\section{Emergence and osteopathy, between physicalism and anti-reductionism}

The idea of a concept of emergence comes from John Stuart Mill who, in A system of logic, considered that the juxtaposition and the interaction of the constituent parts of a living being are not sufficient to explain the properties of the latter.[4] This line of thought dates from the second half of the 19th century and stands as an intermediate proposition between mechanism and vitalism.

In the philosophy of emergence, what emerges is novelty. This novelty is complex and cannot be reduced to the study of its component parts. It has properties of a new kind which did not previously exist in the elements constituting it.

In our work of interpreting osteopathy in terms of the philosophy of emergence, we are drawing on the work of Olivier Sartenaer. He is a graduate in the physical sciences, holds a doctorate in philosophy, and has done a great deal of work on defining the epistemic perimeter of the concept of emergence. Here is the general definition of emergence that he proposes: "Certain natural systems conceived as collections of interrelated entities manifest properties which transcend the simple aggregation of their underlying constituents."[5] In other words, and in accord with the saying in Aristotle's Physics: "the whole is other than the sum of its parts"[6]; this speaks of the irreducibility of the whole to its components, but also sets out the notion of the dependence of an emerging property on the base for supervenience from which it emerges.

I continue the definition: "Such natural systems possess properties described as emergent in the sense that their existence cannot be extrapolated or anticipated on the basis of knowledge, however exhaustive, of the properties of the parts of the system." [5] This is the second essential condition for grasping an emergent property, namely the notion of novelty.

Emergence could also be formulated as an empirical relation between two relata... namely, an "emergent phenomenon" E, and its basis for its emergence B, which is such that we simultaneously find the following two ideas called "dependency" and "novelty":

- (Dependency): E is dependent on - or determined by $-\mathrm{B}$

and yet, in spite of this,

- (Novelty): E is new - or autonomous - with respect to $\mathrm{B}$.

For a phenomenon to be considered emergent it must meet several criteria. Supervenience, holism and topdown causation are the three criteria defined by $O$. Sartenaer [5] based on the definition we gave above.

Supervenience is the formal definition of the dependence of the macro-level with regard to the microlevel. This concept of supervenience presupposes a monism of substances and a continuity on the level of physicality between the emerging phenomenon and its substrate. However, substance monism is an unprovable metaphysical option, as is dualism. We have therefore chosen to accept it and recognize it as being valid in the 
case of osteopathy. Supervenience is the formal definition of the necessary presence of fertile ground (of a substrate), in this case the patient-therapist pairing, so that novelty - the emerging phenomenon - can occur: for example, a certain osteopathic perception.

This is to say that for there to be emergence, there must be matter, and that the emergent phenomenon must be able to arise in the continuity of this matter.

I would therefore like to point out that in our example we are dealing with the patient-therapist pair as interacting material bodies, on which the emerging phenomenon of osteopathic perception depends. This pair is indeed formed of matter to the extent that these are two bodies interacting in the same space-time framework, and the osteopathic perception born as a result of relating the elements of the system is accepted as being expressed within this matter.

Holism is the arising of at least one new property not included in one of the underlying parts. This property is of course a systemic one, meaning that it depends on bringing the elements of the system into relationship, allowing for emergence. And this systemic property is of a new kind compared to the properties of its constituent parts. For example, neither the carbon atom nor even the amino acid molecule are alive. But the cell is [7]. (Here we are talking about life as being an emerging property.) In the case of osteopathic perception, it also respects the criterion of holism in the sense that it did not previously exist in one of the parts of the patient-therapist pair. It is neither present in the therapist in isolation, nor in the patient on her own. There is a capacity for feeling in every living being, but this is not osteopathic perception. Perception is not only noticing a sensation: it is also the act of thinking of what is felt (consciously or not). This action can only be performed in the presence of the osteopath and the patient. Although an osteopath is able to think what he feels without a patient being present, this is not perception. He could imagine it and think of a possible representation of the patient he is going to consult, but this is not osteopathic perception, it is imagination. The criterion of holism is thus indeed respected, as the osteopathic perception of the patient by the therapist is only present as such in the context of the patient-therapist duo.

Top-down causation is the capacity of a complex system to act on and even modify its parts and components, both in terms of their structure and their nature. Returning to the example of the cell, we can say that it is composed, among other things, of lipids, amino acids, etc., and is capable of acting on its own components. It can bring other molecules into its system and change their nature, and this while retaining a relative autonomy of its identity as a cell (for example, a red blood cell remains a red blood cell whether it has attached oxygen or not). This condition of top-down causation is also respected for several reasons in the context of osteopathic perception. In fact, perception is indeed an action which implies the bringing into relationship of several real elements. And an action, by definition, modifies reality, (and I see this on a daily basis: the patient's tissues already begin to change at the very moment when I put my hands on her). The perceptual act in itself will thus indeed modify the part of the system constituted by the patient. On the other hand, the therapist who perceives the patient is no longer the same when he perceives her, compared to how he was before the session. This perceptual act effectively constitutes a new memory within the therapist's experience, which modifies his central nervous system and which can potentially influence his future consultations. There is therefore top-down causation in the osteopathic perceptual phenomenon.

This is an example of the work that we are endeavouring to carry out at the moment within the E.C.O. group. We are currently working to characterize potential loci of emergence and to verify their consistency with the criteria proposed by O. Sartenaer. This work is being carried out by analyzing clinical cases that relate osteopathic experience. In order to situate this approach within a methodology that is usable despite the subjective nature of the osteopathic experience, we have chosen to make use of the explanatory interviews by Pierre Vermersch.[10]

\section{Questions and limits}

The concept of emergence is therefore rather attractive in terms of allowing us to define an epistemology of osteopathy. We do however take note of the criticisms raised by this concept of emergence, and we are currently working with the philosopher $P$. Gagnon on the question of the untenability of nonreducible physicalism, which according to the American metaphysician Jaegwon Kim constitutes the prime conceptual objection to the possibility of emergence. [8]

According to J. Kim, emergence is not tenable because the coexistence of supervenience and the topdown causation of which we spoke earlier would either imply that certain events are systematically overdetermined in causal terms (in which case there is no emergence), or else that the causal closure [8-9] of the physical world can be broken ${ }^{1}$.

O. Sartenaer offers a preliminary response [9] to the critique of $\mathrm{J}$. Kim, and we are continuing our reflection in order for our work not to be trapped within a metaphysical impasse.

\section{Work in progress and perspectives}

The elaboration of a new model that accepts the emergent nature of osteopathic practice and the singular perception of which osteopaths are the subjects would make possible:

- The development of suitable methodological and pedagogical tools, particularly with regard to learning the systemic and holistic dimension of osteopathy. But also, with regard to learning the singular quality of being which allows osteopathic perception to emerge.

- Improvement of the understanding that osteopaths have of their own practice, by giving them mental tools

\footnotetext{
1 The causal closure of the physical world postulates that each physical event has a sufficient physical cause.
} 
allowing complexity and rationality to be brought into dialogue in an open system that accepts that multiple rationalities exist.

- The acknowledgement that in the practice of healthcare, the meeting between two beings can create the conditions of potential permitting the appearance of novelty, which can take the form of a perception, of a modification of one of the systems. The acknowledgement that this novelty, being unpredictable and irreducible, cannot be the object of a study by the experimental sciences as we know them today.

- Support for initiatives that value the subjectivity of our practice while creating inter-subjectivity in order to build commonalities around the sharing of osteopathic experience, particularly in therapist supervision groups. This would simultaneously strengthen the ethical dimension of the osteopathic care relationship.

\section{Conclusion}

I would like to conclude by recalling that this presentation does not claim that reductionism must be abandoned by osteopaths, but rather that it must be systematically embedded within a broader reflection. As E. Morin suggests, complex thought needs to distinguish and join at the same time, in a single operation.[11] In this way osteopathy will be able to continue to enrich itself with each new scientific discovery, while accepting that experimental science may perhaps never be able to account for what is really occurring in our practice.

The meeting between patient and osteopath requires that the therapist be present without being centered on himself, but being aware of the perceiving self. The originality of osteopathy lies in offering a discipline that accepts the individual who practises it in the entirety of her being. In proposing perception as the main investigative tool, osteopathy immediately accepts that there are as many forms of osteopathy as there are perceiving osteopaths. This richness must be safeguarded by refusing to make osteopathy "non operator-dependent".

\section{References}

1. J.M. Gueullette, L'ostéopathie, une autre médecine, Rennes, PUR, 70, 59-68, 181, 130 (2014).

2. World Health Organization, Benchmarks for Training in Osteopathy, 1 (2010).

3. W. Salem, Y. Lepers, Vers une ostéopathie progressiste, Mains libres, 3, 4-5 (2018).

4. J.S. Mill, De la composition des causes (Of the Composition of Causes), Paris, Librairie Philosophique de Ladrange, $405-414$ (1866).

5. O. Sartenaer, Définir l'émergence, Revue des Questions Scientifiques, 181-3, 372 (2010).

6. Aristote, La Métaphysique, 2, Paris, Vrin, 474 (1986).

7. F. Revol, La nouveauté dans l'histoire de la nature, Paris, Vrin, 218 (2015).
8. J. Kim, Physicalism, or Something Near Enough, Princeton, Princeton University Press, chap.2 (2005).

9. O. Sartenaer, Qu'est-ce que l'émergence, Paris, Vrin, 56-64 (2018).

10. P. Vermersch, L'entretien d'explicitation, Paris, ESF (2010).

11. E. Morin, Introduction à la pensée complexe, Paris, Seuil, 103-104 (2005). 